Sociologie et sociétés

\title{
Épistémologies du Sud et militantisme académique
}

Entretien avec Boaventura de Sousa Santos, réalisé par

Baptiste Godrie

\section{Southern Epistemologies and Academic Activism}

Interview with Boaventura de Sousa Santos, conducted by

Baptiste Godrie

Volume 49, numéro 1, printemps 2017

Injustices épistémiques

Epistemic injustices

URI : https://id.erudit.org/iderudit/1042809ar

DOI : https://doi.org/10.7202/1042809ar

Aller au sommaire du numéro

Éditeur(s)

Les Presses de l'Université de Montréal

ISSN

0038-030X (imprimé)

1492-1375 (numérique)

Découvrir la revue

\section{Citer ce document}

(2017). Épistémologies du Sud et militantisme académique : entretien avec Boaventura de Sousa Santos, réalisé par Baptiste Godrie. Sociologie et sociétés, 49(1), 143-149. https://doi.org/10.7202/1042809ar d'utilisation que vous pouvez consulter en ligne. 


\title{
$\geqslant$
}

\section{Épistémologies du Sud et militantisme académique}

\author{
Entretien avec Boaventura de Sousa Santos, \\ réalisé par Baptiste Godrie
}

Quels sont les éléments de votre parcours intellectuel de chercheur en sciences sociales qui vous ont amené à poser un regard critique sur l'épistémologie eurocentriste hégémonique?

Au début des années 1970, dans le cadre de mon doctorat à l'Université Yale, j'ai mené des recherches de terrain sous la forme d'observations participantes. Je vivais dans une favela de Rio de Janeiro et discutais souvent et longuement avec les autres habitants de ces quartiers, des sujets les plus divers. Même si la plupart d'entre eux ne savaient ni lire ni écrire, ils possédaient un bon sens extraordinaire et un discernement remarquable. Entre cette sagesse, à la fois humble et subtile, et les connaissances scientifiques extrêmement pointues, restreintes et présomptueuses de mes professeurs de Yale, quel contraste! Par ailleurs, différents mouvements de protestation secouaient alors les États-Unis: manifestations contre la guerre au Vietnam, Black Panther... Toutes ces turbulences politiques remettaient en cause la science sociale structuro-fonctionnaliste parsonienne, axée exclusivement sur l'ordre et le consensus, et, d'une manière plus générale, la science positiviste. Ces circonstances ont induit chez moi deux réorientations épistémologiques et théoriques. Constituant deux facettes d'une même expérience existentielle, elles ont émergé presque simultanément; elles se sont toutefois développées à des époques différentes et selon des rythmes distincts. S’inscrivant dans le cadre de l'épistémologie eurocentriste, la première consistait à repenser son caractère hégémonique (positiviste) pour affirmer la pluralité interne de la science moderne du point de vue méthodologique, théorique et épistémologique. Mon ouvrage intitulé Cognitive Justice in a Global World (Lexington Books, 2007) rend compte de cette réflexion que j'ai menée sur une vingtaine d'années. En ce qui concerne ma deuxième 
réorientation, le dernier chapitre de mon livre Toward a New Common Sense (Routledge, 1995; version française: Vers un nouveau sens commun juridique, LGDJ, 2004) l'annonçait déjà en préconisant trois nouveaux types d'apprentissages: apprendre que le Sud existe; apprendre à aller au Sud; apprendre du Sud. Cette nouvelle approche commandait de dépasser complètement le cadre de référence eurocentriste pour prendre acte de la validité épistémologique intrinsèque de modes d'acquisition des connaissances ne s'inscrivant pas dans la démarche scientifique. En d'autres termes, il s'agissait de reconnaître la pluralité externe des modes d'acquisition des connaissances. Sur les plans sociologique et existentiel, cette deuxième réorientation doit autant à mes recherches universitaires qu'à mon militantisme politique international. D'une part, j'ai mené des recherches dans plusieurs pays hors Europe (parmi mes ouvrages publiés en anglais, voir par exemple: Democratizing Democracy, 2005; Another Production is Possible, 2006; Another Knowledge is Possible, 2007; et Voices of the World, 2010; tous aux éditions Verso). D'autre part, j'ai commencé à participer très activement au Forum social mondial en 2001 et je suis devenu un militant engagé de l'action politique intermouvement (The Rise of Global Left: the World Social Forum and beyond, Zed Books, 2006). C’est ainsi que j'ai commencé à travailler sur les épistémologies du Sud.

\section{Quel est l'objet de cette épistémologie pluraliste que vous avez développée?}

Les épistémologies du Sud s'intéressent à la production et à la validation des connaissances qui émergent au fil des actes de résistance des groupes sociaux soumis à une injustice, une oppression ou une destruction systématique du fait du capitalisme, du colonialisme ou du patriarcat. L'ensemble, vaste et diversifié, de ces expériences constitue ce que j'appelle le Sud anti-impérialiste. Il n'est pas défini par la géographie: c'est un Sud épistémologique formé d'une multitude de sous-ensembles. Chacun d'eux est un Sud épistémologique en soi et se compose de connaissances produites dans le contexte des luttes contre le capitalisme, le colonialisme et le patriarcat. Ces connaissances se forgent dans tous les lieux où de telles luttes existent, dans le Sud comme dans le Nord géographiques. Le but des épistémologies du Sud est de permettre aux groupes sociaux opprimés d'élaborer des représentations du monde qui leur ressemblent, qui rendent compte de leurs réalités, c'est-à-dire de représenter le monde comme leur appartenant, de s'approprier le monde, car c'est à cette condition seulement qu'ils pourront le changer selon leurs propres aspirations.

La plupart de ces modes de production et d'acquisition des connaissances sont fortement arrimés aux pratiques qui les ont vus naître. Les désigner comme épistémologies constitue un acte d'occupation philosophique (dans le sens d'Occupons Wall Street), car c'est parce que l'Occident considérait la validation des connaissances comme une pratique distincte de toutes les autres que l'épistémologie s'y est constituée en tant que philosophie première.

Si elle favorise les luttes anticapitalistes, anticolonialistes et antipatriarcales, la science moderne a tout à fait sa place dans les épistémologies du Sud — mais uniquement à titre de mode de production de connaissances parmi d'autres, et seulement 
dans la mesure où elle interagit avec ces autres connaissances pour constituer ce que j'appelle une «écologie des savoirs» : un processus d'interpollinisation cognitive visant à renforcer les luttes.

Dans mon projet de recherche le plus récent (Alice: Espelhos estranhos, lições imprevistas/Strange Mirrors, Unsuspected Lessons, financé par le Conseil européen de la recherche en 2011-2016, www.alice.ces.uc.pt), nous avons pris grand soin d'ancrer nos travaux et notre militantisme politique dans les épistémologies du Sud. On trouvera les assises théoriques de ce projet dans The End of a Cognitive Empire: the Coming of Age of the Epistemologies of the South (Duke University Press, à paraitre en 2018).

\section{Vous dites que la connaissance peut engendrer l'ignorance et l'invisibilité. Pourriez- vous préciser votre «sociologie des absences»?}

Les épistémologies du Sud reposent sur l'idée que nos sociétés sont divisées par une ligne de fracture abyssale entre les formes métropolitaines et les formes coloniales de la sociabilité. La sociologie des absences cartographie cet abysse. Elle décrit les mécanismes par lesquels ce décrochement rejette certaines formes de sociabilité dans l'inexistence, dans l'invisibilité radicale, dans le négligeable et l'insignifiant. En rendant visibles les exclusions non abyssales (celles de son flanc métropolitain) et en occultant les exclusions abyssales (celles de son flanc colonial), le colonialisme historique a été le principal architecte de cette fracture. La sociologie des absences analyse les processus par lesquels le colonialisme (de pouvoir, de connaissance ou d'être) continue, conjointement avec le capitalisme et le patriarcat, à produire des exclusions abyssales, c'est-àdire à reléguer certains groupes et certaines formes de vie sociale dans l'inexistence, l'invisibilité, l'infériorité radicale ou la dangerosité radicale, en un mot, à les définir comme négligeables ou menaçants.

Un exemple parmi d'autres. À la fin du XIx ${ }^{\mathrm{e}}$ siècle, l'Europe tout entière célébrait l'avènement du droit du travail et se réjouissait des visées protectrices de ces lois et règlements conçus pour neutraliser, au moins en partie, l'inégalité de pouvoir entre employeurs et employés. Ce qu'elle ne disait pas, c'est qu'à cette même époque, dans les colonies, le droit du travail était une branche du droit criminel, qu'il était le droit de l'esclavage et du travail forcé. Ce qui restait aussi cantonné dans son angle mort, c'est que ce droit du travail invisible, ce droit de l'esclavage et du travail forcé, constituait une condition sine qua non de l'avènement du droit du travail européen et de ses visées protectrices.

À ce propos, la connaissance scientifique étant parfois, sinon toujours, un acte de violence (qui réduit au silence, qui s'approprie, qui rejette dans l'invisibilité), serait-il possible d'envisager un savoir scientifique qui ne soit pas intrinsèquement violent? Comment décririez-vous ces manières anti-hégémoniques de produire le savoir?

Pour les épistémologies du Sud, la connaissance scientifique n'est pas violente. Elle est au contraire précieuse, dans la mesure où elle satisfait à deux conditions. Premièrement, elle doit s'élaborer par des méthodologies non extractivistes, dans 
l'optique de savoir avec l'autre, et non sur l'autre ou à propos de l'autre. Deuxièmement, l'utilité de la connaissance scientifique pour les luttes sociales ne dépend pas tant de cette science elle-même que de leur adéquation par rapport au contexte dans lequel elles s'inscrivent, c'est-à-dire l'écologie des savoirs qui se tisse au fil des luttes pour les renforcer.

\section{Pour en revenir à l'idée qu'il ne peut pas y avoir de justice sociale mondiale sans justice cognitive mondiale, pourriez-vous préciser l'interaction entre connaissance et justice? Qu'est-ce que l'injustice épistémique?}

La conception dominante à l'heure actuelle est celle de la justice comme équité, que propose John Rawls. Elle repose sur une assise épistémologique, le «voile d'ignorance». Or, cette ignorance constitue le versant sombre d'un certain type de connaissance. La «position originelle» amène inéluctablement à «choisir rationnellement» deux principes: pour que les intérêts fondamentaux des citoyens libres et égaux soient bien servis, tout le monde doit bénéficier des mêmes libertés et droits de base; mais tout le monde doit bénéficier aussi d'une juste égalité des chances en éducation et en emploi. Quoique brillamment formulées, les prémisses ou hypothèses de cette conception n'en sont pas moins eurocentristes, et reposent même sur une vision libérale de l'eurocentrisme: un seul type de connaissances plutôt qu'une pluralité des connaissances; une seule rationalité plutôt qu'une diversité des rationalités; l'individu plutôt que la collectivité; les citoyens plutôt que les citoyens et les non-citoyens ensemble; la société et la nature plutôt que la société en tant que nature; l'immanence et la transcendance nettement séparées l'une de l'autre plutôt que la transcendance au cœur même de l'immanence. Toutes ces hypothèses confirment une conception du monde occidentale et libérale et vont dans le sens de l'épistémologie dominante qui la sous-tend. Elles permettent au Nord mondial capitaliste, colonialiste et patriarcal de représenter le monde comme lui appartenant et de le transformer en fonction de ses intérêts dominants (dits « universels»). Mais cette conception convient-elle vraiment aux gens et aux collectivités qui peuplent la terre?

Pour les épistémologies du Sud, il ne saurait y avoir de justice si les gens et les collectivités ne peuvent pas représenter le monde comme leur appartenant, car elles ne peuvent pas, alors, le transformer selon leurs propres espoirs et aspirations. En d'autres termes, l'épistémologie qui sous-tend la théorie de John Rawls interdit à une écrasante majorité des habitants de cette planète de s'approprier pleinement le monde dans lequel ils vivent, de s'y sentir chez eux. Il leur est par conséquent impossible de faire advenir le monde: c'est au contraire le monde qui leur advient. Les épistémologies du Sud cherchent à mettre un terme à cette aliénation et à cet exil épistémiques. 
Vous préconisez la mise en place d'une écologie des savoirs émancipatrice, cosmopolite et non relativiste. Où trouver actuellement les pratiques émancipatrices de production des connaissances et les savoirs alternatifs? Quels sont les liens entre cet aspect de vos recherches et votre intérêt pour des espaces de réflexion et d'action tels que le Forum social mondial (FSM)?

Les pratiques émancipatrices sont partout! Le problème, c'est que les épistémologies dominantes nous empêchent de les discerner, de les valoriser, de les soutenir, et, plus encore, de nous en inspirer, d'apprendre d'elles. Allant à contre-courant des conceptions dominantes, les pratiques anticapitalistes, anticolonialistes et antipatriarcales sont en butte à des politiques de répression, de diabolisation, d'assimilation et d'exclusion par la cooptation. Les épistémologies dominantes forment aujourd'hui la main invisible des sens communs capitaliste, colonialiste et patriarcal. Le FSM a joué un rôle déterminant parce qu'il a su donner à voir et préfigurer un monde post-abyssal, un monde qui constate la fracture abyssale, mais la dénonce, un monde qui voit et rend visible l'extraordinaire bouillonnement d'innovation sociale, de créativité et de production de connaissances à l'œuvre de l'autre côté de cette faille. Le FSM a montré que nous n'avons pas besoin d'alternatives, mais d'une manière alternative de penser les alternatives.

Où avez-vous été le plus lu et étudié? Comment vos travaux sur l'épistémologie sont-ils reçus dans les universités hors de ce que vous appelez le «Nord mondial» et même hors des cercles universitaires?

Mes travaux sont très bien reçus dans les mouvements universitaires et sociaux qui dénoncent la banalisation de l'horreur dans la société, la scandaleuse concentration de la richesse et la destruction suicidaire de la nature. Ils sont particulièrement bien reçus dans les groupes qui adhèrent aux trois idées fondatrices des épistémologies du Sud en tant qu'épistémologie de la politique. Premièrement, contrairement à ce que les processus d'indépendance et les guerres et luttes de libération ont pu laisser penser, la domination moderne occidentalocentrée s'appuie sur trois modes d'oppression: le capitalisme, le colonialisme et le patriarcat. Deuxièmement, l'eurocentrisme divise les groupes sociaux anti-domination selon leurs luttes particulières respectives: certains combattent le capitalisme; d'autres, le colonialisme; d'autres encore, le patriarcat. Troisièmement, tant que ces groupes n'intégreront pas toutes les formes de domination à leurs luttes particulières contre l'une ou l'autre d'entre elles, un autre monde ne sera pas possible.

À ma grande surprise — et à ma plus grande joie —, mes travaux intéressent enfin les enseignants — des universités, mais aussi des niveaux primaires et secondaires qui ne veulent pas ou plus former des conformistes, souvent incompétents, mais aspirent à former plutôt des rebelles compétents. 
En tant que producteurs de connaissances légitimés par le système dominant et engagés dans des carrières individualistes et dans une course incessante à la publication, les chercheurs universitaires contribuent aussi à cette hiérarchisation des savoirs. Pour vous, quel devrait être notre rôle de chercheurs en sciences sociales dans la production des connaissances?

Pour être un chercheur en sciences sociales post-abyssal, il faut avoir un pied dans la vie universitaire et l'autre dans le militantisme politique. Il faut privilégier les méthodologies non extractivistes - savoir avec l'autre, et non sur l'autre. Il faut pouvoir renoncer au confort des institutions canoniques et à celui du partage superficiel, hors corporéité, des expériences, mais aussi savoir résister à la tentation de s'imposer comme un étincelant intellectuel d'avant-garde pour se consacrer si nécessaire à un travail intellectuel de fond, plus discret peut-être. Il faut par ailleurs être assez humble et ambitieux pour s'intéresser concrètement aux connaissances non scientifiques dans l'optique de contribuer à l'écologie des savoirs. Il faut enfin prendre deux risques: le premier, participer aux luttes sociales dans un contexte d'intensification de la criminalisation de la protestation; le second, puiser sa propre légitimation, du moins en partie, dans le succès des luttes contre l'oppression et non plus seulement dans cette obsession bibliométrique des institutions qui mène, dans le meilleur des cas, à l'abêtissement et à l'inutilité, et dans le pire, à la trahison.

\section{À l'avenir, quelles seront, selon vous, les pistes de recherche les plus stimulantes dans les domaines des inégalités et de la production des connaissances?}

Les épistémologies du Sud ne fournissent pas de recettes, et leur efficacité ne se mesure pas uniquement à la qualité intellectuelle des travaux qu'elles suscitent. Elles proposent une alternative épistémologique, méthodologique et pédagogique associée à un engagement plus ferme à l'égard d'une transformation sociale progressiste contre le capitalisme, le colonialisme et le patriarcat. Les épistémologies du Sud sont aussi un cri d'alerte, une exhortation à rompre avec cette trajectoire désastreuse qui est celle des connaissances en sciences sociales depuis une centaine d'années. Au début du xx $x^{\mathrm{e}}$ siècle, les sociétés métropolitaines se trouvaient aux prises avec d'importants problèmes sociaux causés par la première révolution industrielle, la fameuse «question sociale». Elles ne savaient pas grand-chose de ces problèmes, inédits et nombreux, auxquels elles se heurtaient. Les sciences sociales ont alors émergé et mission leur a été donnée de résoudre la question sociale. Aujourd'hui, un siècle plus tard, nous avons accumulé une quantité astronomique de connaissances en sciences sociales — avec quel résultat? Les huit hommes les plus riches du monde possèdent plus que la moitié la plus pauvre de la population planétaire, c'est-à-dire 3,5 milliards de personnes! Et rien ne se passe, parce qu'il semble n'exister aucune alternative à ce modèle.

Les épistémologies du Sud supposent une réforme en profondeur de nos centres de recherche et même une refonte de nos universités, qui prennent de plus en plus des allures d'entreprises commerciales. La mise en place d'une véritable écologie des savoirs implique une inversion du processus de diffusion des connaissances: au lieu de 
les transférer de l'université vers la société, il s'agit maintenant d'apporter les connaissances non scientifiques de l'extérieur de l'université à l'intérieur de ses murs pour favoriser les dialogues et la traduction interculturelle, et renforcer ainsi les luttes sociales contre la domination. 